\title{
Development of Quad Copter Based Pesticide Spraying Mechanism for Agricultural Applications
}

\author{
Sadhana $B^{1}$, Gourav Naik ${ }^{2}$, Mythri R $\mathbf{J}^{3}$, Puneeth G Hedge ${ }^{4}$, Shyama Kirana Sharma $B^{5}$ \\ Assistant Prof, Dept. of ISE, CEC Benjanapadavu, Mangalore, India \\ Student, Dept. of ISE, CEC Benjanapadavu, Mangalore, India ${ }^{2,3,4,5}$
}

\begin{abstract}
Indian agriculture needed production as well as protection materials to achieve high productivity. Fertilizer and chemical frequently needed to kill insects. The WHO (World Health Organization) estimates there are more than 1 million pesticide affective cases in every year. In that around one lakh deaths in each year, especially due to the pesticides sprayed by human being. The pesticide affects the nervous system and also leads to disorders. A Remote Controlled Unmanned Aerial Vehicle (UAV) is used to spray the Pesticide. The vertical take-off and landing quad copter is used to spray the pesticide. This paper describes the development of quad copter UAV and the sprayer module, discusses the integration of sprayer module to quad copter system. Total payload lift off weight of quad copter is $1 \mathrm{~kg}$.
\end{abstract}

Keywords: Unmanned Aerial Vehicle, drones, quadcopter, manual spraying, pesticide sprayer, pesticide spraying mechanism.

\section{INTRODUCTION}

The potential health effects of pesticides may include asthma, allergies, and hypersensitivity, cancer, hormone disruption and problems with reproduction and fatal development and many more health issues.

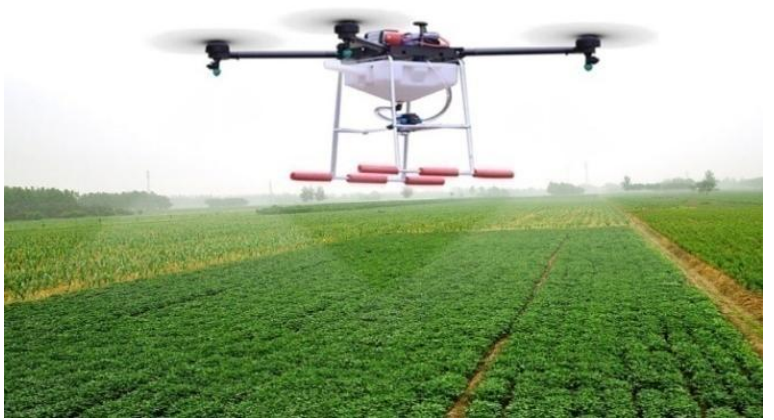

Fig 1: Pesticide spraying mechanism

The quadcopter was chosen for this project because of two benefits like its high stability and more lifting power. The control of quadcopter is easier while compared with the helicopter model or vehicles. Some applications of quadcopter include are Search and Rescue, Police, Code Enforcement/Inspections, Emergency Management, Fire, Defence Surveillance, Border security, etc.

\section{LITERATURE SURVEY}

What "drones" means to Kunde and the growing number of farmers like him is simply a low-cost aerial camera platform either miniature fixed-wing airplanes or, more commonly, quad copters and other multiplied small helicopters. These aircraft are equipped with an autopilot using GPS and a standard point-and-shoot camera controlled by the autopilot; software on the ground can stitch aerial shots into a high- $\neg$ resolution mosaic map. Whereas a traditional radio--controlled aircraft needs to be flown by a pilot on the ground, in Kunde's drone the autopilot (made by my company, 3D Robotics) does all the flying, from auto takeoff to landing. Its software plans the flight path, aiming for maximum coverage of the vineyards, and controls the camera to optimize the images for later analysis [1]

Remote sensing by Unmanned Aerial Vehicles (UAVs) is changing the way agriculture operates by increasing the spatial-temporal resolution of data collection. MicroUAVs have the potential to further improve and enrich the data collected by operating close to the crops, enabling the collection of higher spatial-temporal resolution data. In that paper, they presented a UAV-mounted measurement system that utilizes a laser scanner to compute crop heights, a critical indicator of crop health. The system filters, transforms, and analyzes the cluttered range data in real-time to determine the distance to the ground and to the top of the crops. We assess the system in an indoor testbed and in a corn field. Our findings indicate that 


\section{International Journal of Innovative Research in Electrical, Electronics, Instrumentation and Control Engineering NCAEE 2017 \\ National Conference on Advances in Electrical Engineering \\ NMAM Institute of Technology, Nitte \\ Vol. 5, Special Issue 2, April 2017}

despite the dense canopy and highly variable sensor popular choice of battery for a QuadCopter is the 3SP1 readings, we can precisely fly over crops and measure its batteries which means three cells connected in series as height to within $5 \mathrm{~cm}$ of measurements gathered using one parallel, which should give us $11.1 \mathrm{~V}$. current measurement technology[2].

\section{HARDWARE DESCRIPTION}

\section{Arduino Uno}

The Arduino Uno is a microcontroller board based on the ATmega328. It has 14 digital input/output pins (of which 6 can be used as PWM outputs), 6 analog inputs, a 16 $\mathrm{MHz}$ ceramic resonator, a USB connection, a power jack, an ICSP header, and a reset button. It contains everything needed to support the microcontroller; simply connect it to a computer with a USB cable or power it with a AC-to-DC adapter or battery to get started.

\section{BLDC $(1800 \mathrm{Kv})$}

This is a brushless (BLDC) out runner motor specifically made for quadcopters and multirotor. It is a $1800 \mathrm{kV}$ motor. It provides high performance, super power and brilliant efficiency. The 30A ESCs can be used to drive the motor.

\section{ESC}

ESC is used to control BLDC motor. It takes signal from microcontroller and breaks into 3 parts and sends it to the BLDC motor. We would require 4 ESCs as we are using 4 BLDC motor. The ESC generates three high frequency signals with different but controllable phases continually to keep the motor turning. The ESC is also able to source a lot of current as the motors can draw a lot of power.

\section{MPU-6050}

The InvenSense MPU-6050 sensor contains a MEMS accelerometer and a MEMS gyro in a single chip. It is very accurate, as it contains 16-bits analog to digital conversion hardware for each channel. Therefor it captures the $\mathrm{x}, \mathrm{y}$, and $\mathrm{z}$ channel at the same time. The sensor uses the I2C-bus to interface with the Arduino.

\section{Radio receiver}

This receives $2.4 \mathrm{GHz}$ signals coming from the transmitter side. It has got 6 independent channels to receive the signal from the transmitter and then send the signal to the microcontroller for further processing. Its current consumption is less than $40 \mathrm{~mA}$ and works on 5 volt power supply.

\section{LIPO Battery}

Lithium batteries are the preferred power sources for most electric modelers today. They offer high discharge rates and a high-energy storage/weight ratio. However, using them properly and charging them correctly is no trivial task. LiPo battery can be found in a single cell $(3.7 \mathrm{~V})$ to in a pack of over 10 cells connected in series (37V). A

\section{PROPOSED METHODOLOGY}

The quadcopter system is first switched on and this in turn causes the quadcopter to switch on. The joystick is kept at position zero. Once it is at position zero the gyroscope starts calibration and the indication light turns on, later blinks. After the light turns off the joystick is moved to position start 1 . The throttle then is moved. After that even if there is no input from joystick the readings from the gyroscope is taken and the quadcopter moves according to the readings of the gyroscope. When quadcopter moves to the desired location, the sprayer module can be turned on. After that the joystick is moved back to start 0 position. Once the joystick is in start 0 the motor gets turned off. Thus, the system gets turned off.

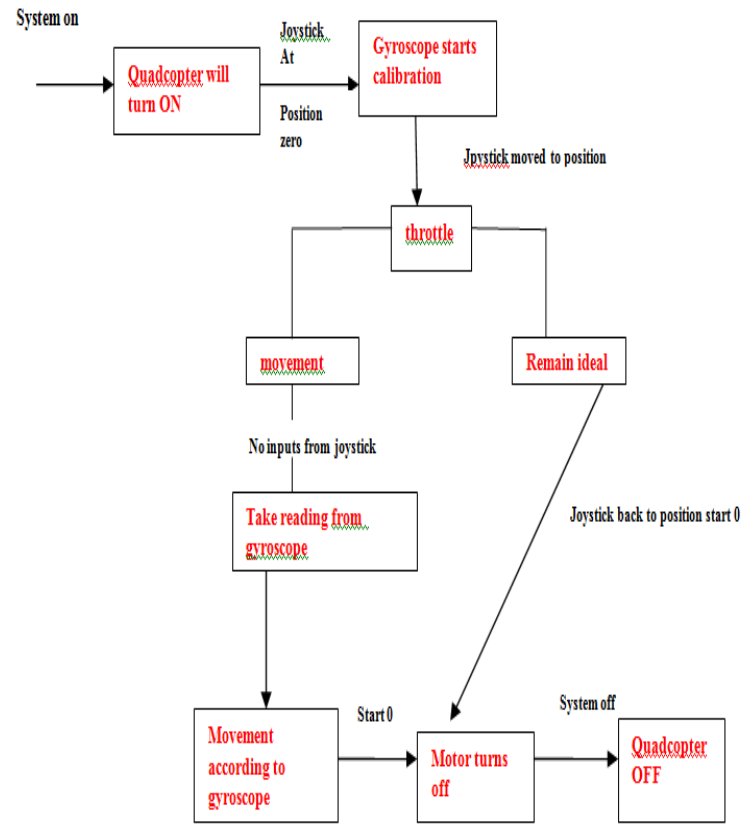

Fig 2: Working of quad copter.

Figure 3 shows the block diagram of pesticide spraying mechanism to be integrated with quadcopter.

Transmitter side:

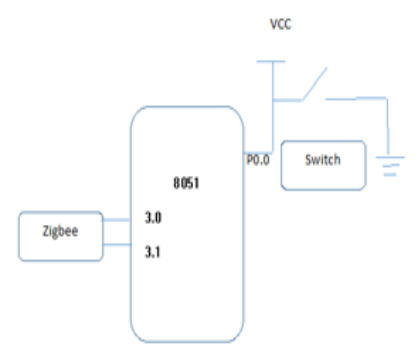


Receiver side:

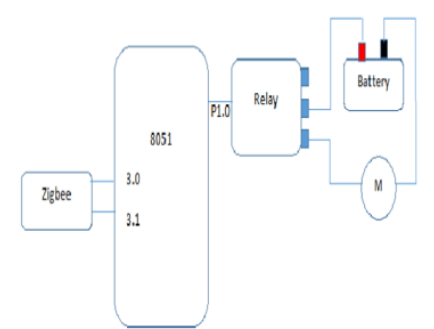

Fig 3: Block diagram of pesticide spraying module

For the pesticide spraying mechanism we use pesticide tank of capacity $180 \mathrm{ml}$, submersible dc motor pump, ZigBee module, 8051 microcontroller, relay, pipes fitted to $\mathrm{T}$-split and mini nozzles. The working of spraying module is described in Figure 4.

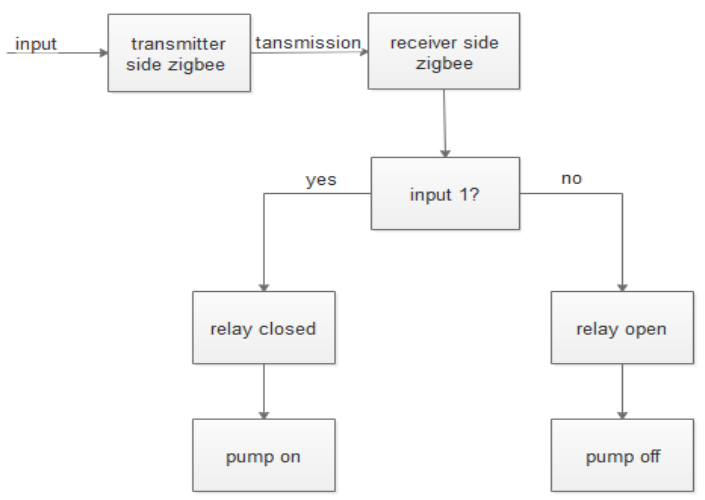

Fig 4: working of spraying module

The input from the ground station microprocessor 8051 is transmitted to ZigBee module of transmitter side, which in turn transmits it to receiver side $\mathrm{ZigBee}$ and is passed onto 8051 microprocessor of receiver side. Depending on the input the relay remains open or closed. If the relay is open the pump is turned off else on.

\section{EXPERIMENTAL RESULTS AND CONCLUSON}

This method can be used in all situations, especially in the places where labours are hard to find. It has many advantages that include hastening the spraying process of pesticide thereby reducing the causalities due to pesticide exposures and hence prevents the encounters with the poisonous snakes like viper and cobra which are regularly found in our agricultural fields. Environmental pollution can be reduced when it sprayed from lower altitude. The spraying time of pesticides is dependent on the quantity of pesticide to be sprayed. For example, for $1000 \mathrm{ml}$ of pesticides, spraying time is around 5 minutes. If we want to increase the quantity of pesticide to be sprayed, the weight lifting capacity of the quadcopter must be increased. This is done by choosing higher specification of BLDC i.e. more than $1000 \mathrm{rpm} / \mathrm{kV}$. In future, the system can be made autonomous by replacing the Arduino Uno with advanced flight controllers. Google way points can be used for flight path.

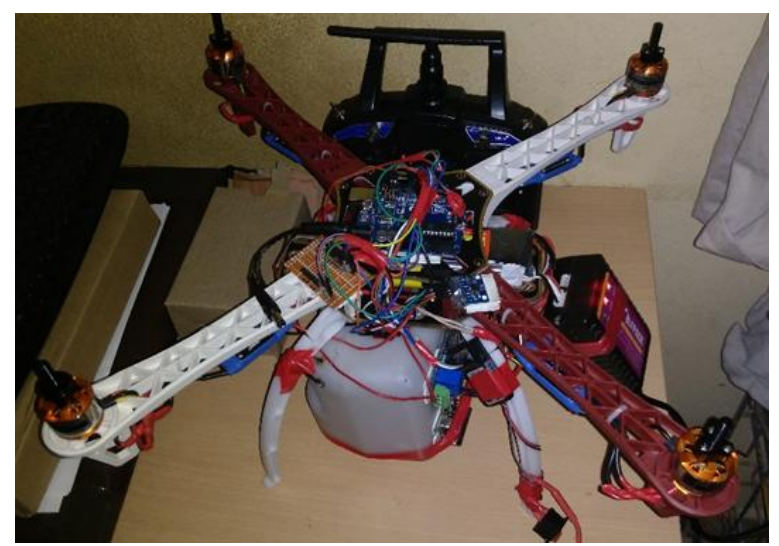

Fig 5: Quad copter based pesticide spraying system

\section{ACKNOWLEDGMENT}

We are thankful to all the authors of the papers those we referred and for providing the necessary opportunities for the preparation of the paper.

\section{REFERENCES}

[1] MIT Technology Review," Agricultural Drones. Relatively cheap drones with advanced sensors and imaging capabilities are giving farmers new ways to increase yields and reduce crop damage".

[2] D. Anthony, S. Elbaum, A. Lorenz, C. Detweiler, "On Crop Height Estimation with UAVs", 2014 IEEE/RSJ International Conference on Intelligent Robots and Systems.

[3] C. Zhang, J. M. Kovacs," The application of small unmanned aerial systems for precision agriculture: a review", Precision Agriculture, Springer, 2012.

[4] F. G. Costa, J. Ueyama, T. Braun, G. Pessin, F. S. Osorio, P. A. Vargas, "The Use of Unmanned Aerial Vehicles and Wireless Sensor Network in Agriculture Applications", 2012IEEE International Geoscience and Remote Sensing Symposium, 2012.

[5] Colomina, P. Molina, "Unmanned aerial systems for photogrammetry and remote sensing: a review", ISPRS Journal of Photogrammetry and Remote Sensing, June 2014. 